\title{
ENFERMEMENT CARCÉRAL, IMAGINAIRE ET PATHOLOGIE DE L'ADAPTATION
}

Jérôme Englebert

ERES | Empan »

$2019 / 2 \mathrm{n}^{\circ} 114 \mid$ pages 22 à 30

ISSN 1152-3336

ISBN 9782749264455

Article disponible en ligne à l'adresse :

https://www.cairn.info/revue-empan-2019-2-page-22.htm

Distribution électronique Cairn.info pour ERES.

(C) ERES. Tous droits réservés pour tous pays.

La reproduction ou représentation de cet article, notamment par photocopie, n'est autorisée que dans les limites des conditions générales d'utilisation du site ou, le cas échéant, des conditions générales de la licence souscrite par votre établissement. Toute autre reproduction ou représentation, en tout ou partie, sous quelque forme et de quelque manière que ce soit, est interdite sauf accord préalable et écrit de l'éditeur, en dehors des cas prévus par la législation en vigueur en France. Il est précisé que son stockage dans une base de données est également interdit. 
SITUATION

\section{DE LA}

CONTRAINTE

\section{Enfermement carcéral,}

imaginaire et pathologie de l'adaptation

Jérôme Englebert

À Jean-Marie Gauthier

« Il semble que l'imagination qui hante à des degrés divers l'esprit de toute créature soit pressée de se séparer d'elle quand celle-ci ne lui propose que l'impossible et l'inaccessible pour extrême mission. Il faut admettre que la poésie n'est pas partout souveraine. »

René Char, Feuillets d'Hypnos, 1946, p. 132.

Il faut bien reconnaître que l'imaginaire est le grand oublié de l'horizon épistémologique des sciences humaines. Selon la conception que nous allons exposer, il s'agit d'une dimension fondamentale de l'être humain, l'une de ses grandes attitudes subjectives. Cette perspective est, selon nous, l'apport le plus fondamental des travaux de Sami-Ali (1970, 1974, 1977, 1980, 1987, 1990, 1997), et c'est à partir des propositions théoriques de cet auteur, enrichies par les études de Sartre (1940) et de Bachelard (1942, 1957), que nous allons étudier ce « grand absent».

La subtilité de l'imaginaire réside dans son interconnexion complexe avec le réel. L'un n'existe pas sans l'autre. Cependant, deux exceptions doivent être citées, avant d'être détaillées par la suite : le rêve (l'imaginaire " pur») et la pathologie de l'adaptation (le réel «pur» que Sami-Ali (1980) appelle « le banal»). L'hypothèse de ce texte sera : l'univers carcéral favorise l'inscription de l'imaginaire dans la négativité, s'inscrit dans le banal. Mais avant de parler de l'absence d'imaginaire, précisons cette «puissance majeure de la nature humaine » (Bachelard, 1957, p. 16) dans toute sa positivité.

Jérôme Englebert, département de psychologie, université de Liège, quartier Agora, place des Orateurs 1 (bât. B 33), 4000 Liège (Belgique). jerome.englebert@ULiege.be

\section{LA « SIMPLICITÉ " DE L'IMAGINAIRE}

Une lecture de l'essai de Sartre (1940) permet de suggérer que l'imaginaire est un phénomène très simple : il s'agirait de tout 
phénomène qui s'éloigne du réel. Cette « grande fonction irréalisante de la conscience » (Sartre, 1940, p. 13), ce moment et ce lieu où s'amenuise la clairvoyance objective au profit de la création subjective. Il s'agit d'une dimension élémentaire ouvrant la voie à la subjectivité : «La réalité imaginaire est de l'ordre du $T u$ avant d'être de l'ordre du Cela» (Bachelard, 1942, p. 139). À travers cette perspective, nous comprenons que recourir à l'imaginaire, et lui donner la place qui lui revient, conduit à anéantir cet idéal impossible - et lui-même irréel-d'objectivité complète en sciences humaines.

Toujours selon ce paradigme de simplicité, l'imaginaire peut être défini comme une fonction permettant au sujet d'appréhender une image sans avoir recours à la perception. La qualité essentielle de l'objet saisi par l'imaginaire, cet irréel, est de «n'être pas là » (Sartre, 1940, p. 242-243). L'objet imagé (c'est-à-dire sa dimension irréelle) jouit d'une temporalité spécifique qui est, en quelque sorte, arrêtée alors que la temporalité de l'objet en tant que réalité continue de se développer : "Il n'est donc nullement prouvé que le temps d'écoulement de la conscience d'image soit le même que le temps de l'objet imagé. Nous allons voir, au contraire [...] que ces deux durées sont radicalement séparées » (ibid., p. 248). L'objet imagé est un intemporel là où l'objet réel qu'il représente s'inscrit dans le temps et dès lors continue d'évoluer et de se transformer. Comme nous l'observions dans d'autres travaux (Englebert, 2013a), le sujet incarcéré garde des souvenirs imagés de la réalité extérieure qui, en tant qu'objets imaginaires, n'évoluent plus, ou évoluent selon la construction irréelle du sujet. À l'inverse, l'objet « réel », extérieur à la prison, auquel le sujet pense (qu'il s'agisse d'un lieu, d'une personne, etc.) continue sa « mutation naturelle » indépendamment du sujet.

Se limiter à considérer l'imaginaire comme une image articulée à un objet réel extérieur et tangible serait erroné. Ce serait faire fi de sa fonction de création. L'imaginaire ne s'agence pas obligatoirement et essentiellement à un objet ancien ou déjà concrètement perçu; il est aussi un acte de créativité et d'invention psychique. L'imaginaire est la source de la projection (Sami-Ali, 1970) et de la construction d'images qui peuvent être indépendantes d'un corrélat réel et concret. C'est cette possibilité de l'imaginaire qui permet à Boris Vian de préciser dans L'écume des jours que « [...] l'histoire est entièrement vraie, puisque je l'ai imaginée d'un bout à l'autre » (Vian, 1947, p. 17).

Enfin, l'imaginaire a également une fonction « troublante » permettant d'éviter la répétition stéréotypée du réel (Sami-Ali, 1980). Le rôle de l'imaginaire est d'interpeller l'homme dans la fonctionnalité et la rentabilité pragmatique : «L'imagination se place dans la marge où précisément la fonction de l'irréel vient séduire ou inquiéter - toujours réveiller - l'être endormi dans ses automatismes » (Bachelard, 1957, p. 17).

\section{RÊVE ET PROJECTION}

Pour Sami-Ali, l'imaginaire pur, c'est le rêve ; c'est-à-dire une situation dans laquelle le temps et l'espace se comportent de façon magique. Le temps du rêve est un temps paradoxal qui permet la réversibilité ; dans le rêve, il est possible de voyager dans le temps, de le remonter. Les limites entre passé, présent et futur sont distendues et ne sont plus hermétiques. Le temps du rêve est circulaire (et non pas linéaire) et, par son principe de réversibilité, ouvre la voie à une confusion entre cause et effet.

L'espace du rêve ne connaît pas, non plus, la contradiction. Il est organisé sur le mode de l'inclusion réciproque, comme des poupées russes qui parviendraient à s'inclure l'une dans l'autre. Cet espace est sans surface, sans profondeur ; il n'a pas besoin de cohérence et le sujet du rêve y vit dans une logique d'indifférenciation à l'autre. Le rêveur y est à la fois l'acteur, l'objet de l'acte et le scénariste ; la topique onirique est singularité et réciproquement totalité (Sami-Ali, 1974). Dans cet espace, il y a équivalence entre contenu et 


\section{L'imaginaire est donc une sorte}

\section{dioutil de résolution}

des difficultés

quotidiennes,

entre adaptation

au monde

et transformation

de celvi-ci. contenant, entre le dedans et le dehors, entre le corps propre et l'espace. On est proche des propositions de Bachelard pour qui la Poétique de l'espace consiste en une étude du lieu qui n'est plus celui de la positivité du géomètre mais celui vécu « avec toutes les partialités de l'imagination» (Bachelard, 1957, p. 17).

L'étude de l'imaginaire nous permet donc de concevoir ces deux variables que sont l'espace et le temps sous un jour nouveau. La temporalité ne peut plus être étudiée sans prendre en compte son corrélatif irréel qu'est le "temps imaginaire ", de même que la spatialité devra dorénavant se comprendre en lui articulant sa possibilité d'« espace imaginaire ».

Parler de l'imaginaire oblige également à parler de la définition originale, proposée par Sami-Ali, du concept de projection. Ce qu'il appelle projection diffère en de nombreux points du concept freudien. Dans De la projection (1970), Sami-Ali élargit considérablement le champ d'application de ce concept : « La projection, loin de se ramener à un mécanisme de défense, coïncide avec la possibilité même que le sujet, en se scindant, crée, en dehors de lui, un monde qui est lui » (Sami-Ali, 1970, p. 137). Mouvement fondateur de la vie psychique, ce mécanisme, non plus de défense mais de création, tend, en établissant des équivalences entre le corps et l'environnement, à constituer la possibilité même de penser (SamiAli, 1970). Cette projection fondamentale est un mode particulier de relation du sujet à son environnement qui condamne l'ensemble du réel sous le joug de l'imaginaire. Cette " projection primordiale » se met en place à ce moment où la réalité externe commence à exister comme différente de soi.

Comme nous venons déjà de fortement le suggérer, il convient de concevoir imaginaire et réel, conscience onirique et conscience vigile, projection et perception comme des dimensions qui ne sont séparables que pour le besoin de l'élaboration théorique. Il y a une réflexivité indivisible entre réel et irréel, un englobement mutuel : « Il ne saurait y avoir de conscience réalisante sans conscience imageante et réciproquement. Ainsi l'imagination, loin d'apparaître comme une caractéristique de fait de la conscience, s'est dévoilée comme une condition essentielle et transcendantale de la conscience. Il est aussi absurde de concevoir une conscience qui n'imaginerait pas que de concevoir une conscience qui ne pourrait effectuer le cogito » (Sartre, 1940, p. 361).

En outre, Sami-Ali et Sartre permettent de comprendre que cette dimension imaginaire ne doit pas être considérée comme un état secondaire au réel qui viendrait simplement troubler l'être dans ses activités subjectives. Il semble, plutôt, que le noyau même de la subjectivité soit enfermé dans cette dialectique subtile : «Tout imaginaire paraît "sur fond de monde", mais réciproquement toute appréhension du réel comme monde implique un dépassement 
caché vers l'imaginaire » (Sartre, 1940, p. 361). L'imaginaire est donc une des grandes attitudes de l'homme et, non pas secondaire, il est le fondement même du sujet et de sa subjectivité. Le mouvement d'inclusion réciproque entre réel et imaginaire est la condition sine qua non de l'édification du sujet pensant car, sans ce subtil dosage, nous sommes soit du côté de la vie onirique avec une subjectivité sans un sujet concret et sans conséquences mondaines, soit du côté du banal - concept pour lequel nous donnerons une définition argumentée ci-après - avec un sujet sans subjectivité s'exprimant dans un monde strictement limité à des conséquences auxquelles le sujet doit se soumettre.

\section{CoRPS ET ALTÉRITÉ}

Une dernière caractéristique essentielle doit encore être précisée à propos de l'imaginaire : son ancrage fondamentalement corporel. Comme souvent, il est intéressant de comprendre l'histoire personnelle de l'auteur pour saisir celle de sa théorie. À cet égard, nous pouvons préciser que Sami-Ali est issu de la mouvance psychanalytique - il était notamment proche de Didier Anzieu -, avec laquelle il a fini par rompre. Pour comprendre sa théorie psychosomatique, il est utile d'observer en quoi celle-ci s'éloigne progressivement de l'épistémologie freudienne pour tendre vers une rupture irrémédiable. Le corps, tel que le conçoit Sami-Ali, ne peut plus se limiter au corps pulsionnel freudien et en cela induit une rupture avec la tradition psychosomatique classique - laquelle conçoit, sur le modèle de la conversion hystérique, que le corps à travers sa symptomatologie doit être interprété selon une grille de lecture symbolique (sexuelle). Le principe, assez simple et facilement reproductible, est d'attribuer aux symptômes ou affections corporels une signification symbolique. L'hystérie constitue l'horizon épistémologique tant de la psychanalyse que des courants psychosomatiques qui en sont issus : «L'hystérisation du corporel marque, en fait, les limites du savoir psychanalytique» (Gauthier, 1993, p. 62). Cela signifie que, dans ces conditions, il est impossible de penser le corps sans en faire le terrain d'expression de désirs inconscients (le retour du refoulé). L'exemple freudien traditionnel est celui du jeune enfant qui voit son bras paralysé par son désir masturbatoire refoulé.

Il faut plutôt, pour suivre Sami-Ali, retourner aux choses mêmes et tendre vers un corps dans sa dimension phénoménale. Bien qu'il semble refuser l'inscription de son travail dans le giron phénoménologique, il est intéressant de repérer les proximités de Sami-Ali avec cet autre penseur du corps, si proche de lui, qu'est Merleau-Ponty. Car pour tous les deux, en plus de s'inscrire dans l'espace et le temps, le corps est, plus qu'une interface entre l'interne et l'externe, le schéma structurant de représentation par excellence (Merleau-Ponty, 1945 ; SamiAli, 1974,1990). En fait, espace et temps, d'une part, et corps vécu, de l'autre, entretiennent une relation d'influence mutuelle : « Il ne faut donc pas dire que notre corps est dans l'espace ni d'ailleurs qu'il est dans le temps. Il habite l'espace et le temps » (Merleau-Ponty, 1945, p. 162). Car, si avoir un corps revient à se situer dans l'espace et le temps, il est aussi un principe radical faisant que c'est le corps qui, grâce à la médiation perceptive, crée les coordonnées spatio-temporelles (Merleau-Ponty, 1945 ; Sami-Ali, 1974, 1990).

Le corps permet au sujet de se repérer dans l'espace en situant toute réalité par rapport à ce corps propre par les coordonnées classiques devant/derrière, à gauche/à droite, en haut/en bas (Sami-Ali, 1990, 2003). La conscience est radicalement charnelle et incarnée et, à l'origine, permet l'inscription dans la spatio-temporalité mondaine autant qu'elle la crée. Le corps propre est la matrice primordiale permettant l'émergence d'une représentation psychique (Merleau-Ponty, 1945 ; Sami-Ali, 1990).

Cette inscription dans le corporel permet de penser l'imaginaire sous une dimension nouvelle, elle lui donne une dimension anthropologique. Cette magie typiquement humaine est loin de n'être qu'une forme de pensée incomplète et dérisoire et permet 
Passé cette phase inaugurale de la mobilisation de limaginaire, le quotidien carcéral, une fois appréhendé, est dominé par le banal et par un réel qui ne se modifie guère et dès lors auquel on ne pent s'adapter. plutôt à l'homme en situation une subtile alchimie qui consiste à s'adapter au monde tout en le transformant. L'imaginaire nous permet, en quelque sorte, d'anticiper et de dépasser les difficultés que nous rencontrons au quotidien pour atteindre le pouvoir de transformation de la contingence. L'imaginaire est donc une sorte d'outil de résolution des difficultés quotidiennes, entre adaptation au monde et transformation de celui-ci; il permet au réel d'être territorialisé par notre subjectivité et à notre subjectivité de tolérer et d'appréhender le réel.

Si nous utilisons le concept de magique pour qualifier le rapport du sujet à son expérience propre de l'imaginaire, nous apprécions avec Sartre, lorsqu'il s'agit de l'imaginaire de l'autre en tant que sujet distinct de moi que je scrute, de parler de sorcellerie : «L'homme est toujours un sorcier pour l'homme et le monde social est d'abord magique » (Sartre, 1939, p. 58). Si, au contact de l'imaginaire, je deviens un magicien capable de transformer le réel, je dois aussi intégrer le fait que l'autre présente également une magie qui m'est plus étrange, ou de laquelle je ne peux prévoir l'expression. Plus qu'un magicien, l'autre est donc un sorcier dans le sens où cet imaginaire présente pour moi, en partie du moins, une certaine opacité (Englebert, 2013b, 2014). Le sujet doit concevoir qu'il n'est pas le seul à être doté de cette force (ce pouvoir magique) et doit intégrer l'autre dans sa situation en tant que sujet mû également par un imaginaire. Une magie apparaissant étrange qui appartient à l'autre et qui sera source pour lui de connaissance du réel et de sa modification, mais également source de connaissance sur moi, en tant que ce que j'apparais à ses yeux, et dès lors, également, de modification de ce que je suis. À travers sa sorcellerie, je dois accepter qu'à ses yeux, l'autre me crée. En ce sens, comme le souligne Sartre, «la véritable limite de ma liberté est purement et simplement dans le fait même qu'un autre me saisit comme autre-objet [...] » (Sartre, 1943, p. 608).

\section{LE BANAL}

Nous devons maintenant nous poser la question de savoir comment cela se passe quand cet imaginaire n'est pas là ou, du moins, envisager la possibilité d'« une infirmité du côté de la fonction de l'irréel [qui] entrave le psychisme producteur " (Bachelard, 1957, p. 16). Cette évocation de la possibilité d'une absence de la fonction irréalisante nous permettra de coller, petit à petit, à la réalité carcérale.

Le banal est, en effet, ce concept qui permet de formuler un amendement à la description anthropologique, en apparence sans ombrage, à laquelle nous avons abouti concernant l'imaginaire et de répondre par l'affirmative à cette question que nous pose Sartre : «Peut-on concevoir une conscience qui n'imaginerait jamais et qui serait tout entière absorbée dans ses intuitions du réel $[\ldots]$ ? » (Sartre, 
1940, p. 344). Sami-Ali décrit ce type de fonctionnement psychique qui présente, selon lui, un refoulement réussi - car il ne connaît pas l'échec et ne tolère pas le retour du refoulé - de l'imaginaire. La première caractéristique d'un fonctionnement psychologique dirigé par le banal est donc l'absence d'imaginaire, de rêve ou de jeu, « comme si tout devait se réduire à un réel extérieur au sujet [...]» (Sami-Ali, 1977, p. 85). La seconde caractéristique du banal est l'adaptation par répétition stéréotypique : « Le banal correspond à un ensemble de règles adaptatives dont l'application aboutit à la reproduction du même à l'intérieur d'un comportement conforme » (Sami-Ali, 1997, p. 84-85). Le banal présente donc un rapport intime à l'adaptation dans le sens où les sujets inscrits dans le banal présentent une adaptation sans faille et psychorigide. Alors que l'imaginaire permettait une adaptation à l'environnement tout en le transformant, le banal ne peut envisager le moindre acte de transformation qui ne viendrait pas de l'extérieur du sujet.

L'exemple classique est un sujet qui concède ne pas rêver, ne pas s'en souvenir ou n'y accorder aucune importance (Sami-Ali, 1980). L'important n'est pas de savoir s'il y a une absence « concrète » ou neurologique de la vie onirique, mais d'observer le rapport subjectif que le sujet porte à sa vie onirique, en particulier, et à sa vie imaginaire, en général. Ce qui caractérise le banal au niveau de l'activité onirique est donc moins l'absence de rêves que le peu d'intérêt pour ces derniers. Ces patients pouvant répéter inlassablement qu'ils rêvent mais que cela ne les concerne pas ou ne les intéresse pas.

Lorsque nous avons essayé d'aborder la vie onirique avec des prisonniers, certains pouvaient ne pas comprendre ce que nous leur demandions, ne pas comprendre l'intérêt que le rêve peut représenter. Depuis notre pratique clinique, nous sommes amenés à penser que le banal est le mode de fonctionnement privilégié de l'activité onirique du détenu. Une étude consacrée à la production quantitative de rêves (identifiés subjectivement par le détenu) que nous avions menée par ailleurs (Englebert,
$2013 a$, p. 289-299) confirmait cette hypothèse en la nuançant légèrement. Nous constations, dans un premier temps, que lors des premières semaines d'enfermement, contrairement à l'intuition qui nous menait, l'imaginaire semblait fortement « excité » et apparaissait comme étant particulièrement utile à la « survie » en prison. Par contre, une fois cette mobilisation passée, au bout de quelques semaines, le recours au rêve semblait diminuer inexorablement. Nous suggérions (et il ne s'agit bien sûr que d'une hypothèse) que le mécanisme onirique tendrait à s'essouffler puisqu'il ne rencontre pas les effets qu'il est censé produire, c'est-à-dire une modification du réel suffisante pour permettre de s'y adapter (on ne déplace pas les murs d'une prison...). Passé cette phase inaugurale de la mobilisation de l'imaginaire, le quotidien carcéral, une fois appréhendé, est dominé par le banal et par un réel qui ne se modifie guère et dès lors, nous l'avons vu, auquel on ne peut s'adapter. Une adaptation impossible donc, sauf au prix de la pathologie de l'adaptation...

\section{LA PATHOLOGIE DE L'ADAPTATION}

Le chemin que nous venons de parcourir à travers l'imaginaire semble nous conduire aux mêmes conclusions que Sartre lorsqu'il observe que " la condition essentielle pour qu'une conscience puisse imager [est] qu'elle ait la possibilité de poser une thèse d'irréalité »" (Sartre, 1940, p. 351). En effet, il semble que, si la condition a priori de l'imaginaire est la possibilité de dépassement du réel, une absence de cette condition inscrit le sujet et son environnement dans le banal et dans un rapport paradoxal au mécanisme d'adaptation. Ces constats nous conduisent en réalité à redéfinir le concept d'adaptation, et il semble bien que ce concept ne puisse être compris correctement si nous ne lui intégrons pas une logique de modification et de transformation de l'environnement. Il semble en effet que le mécanisme d'adaptation puisse se décliner de deux façons. Nous sommes ici au plus proche des propositions de Canguilhem dans son essai sur Le normal et le pathologique lorsqu'il précise : «En fait, il $\mathrm{y}$ a adaptation et adaptation [...]. Il existe une 


\section{Cette pathologie de liadaptation \\ caractérise}

ces situations

de sujets

qui ont réussi

cet «impossible»

qui consiste

à s'adapter

à liunivers carcéral. forme d'adaptation qui est spécialisation pour une tâche donnée dans un milieu stable, mais qui est menacée par tout accident modifiant ce milieu. Et il existe une autre forme d'adaptation qui est indépendance à l'égard des contraintes d'un milieu stable et par conséquent pouvoir de surmonter les difficultés de vivre résultant d'une altération du milieu. [...] En matière d'adaptation, le parfait ou le fini c'est le commencement de la fin des espèces » (Canguilhem, 1966, p. 197).

Il y a donc deux perspectives en matière d'adaptation :

- une hyper-adaptation au réel qui a pour conséquence de conserver un environnement strictement identique et inchangé. L'organisation de l'espace, du temps et des rythmes vient de l'extérieur et n'est pas frappée par la subjectivité du sujet (c'est bien le cas de la vie carcérale). Nous avons alors un espace et pas de territoire, et l'objectivité prime sur la subjectivité ;

- une adaptation à un environnement dans lequel le réel est, en partie du moins, manipulable et «territorialisable ». Cette adaptation, "déstabilisée » par l'imaginaire, parvient à intégrer la nouveauté et le changement. L'équilibre dialectique entre les rythmes produits par le sujet et ceux produits par l'environnement est conservé (voir Englebert et Follet, 2016).

Le mécanisme d'adaptation précisé, venons-en à la «pathologie de l'adaptation » (Sami-Ali, 1980). Il s'agit d'un fonctionnement psychique dominé par la répétition stéréotypée, l'absence d'imaginaire et l'impossibilité d'investir subjectivement le vécu quotidien : « La pathologie de l'adaptation [...] est cette forme particulière de fonctionnement normal dans laquelle les traits de caractère remplacent les symptômes névrotiques ou psychotiques, alors que l'adaptation s'effectue au détriment du rêve et de ses équivalents » (Sami-Ali, 1977, p. 84-85). En ce sens, le banal est une pathologie de l'adaptation qui consiste en une adhésion parfaite du sujet à son environnement. Ce «collage» ne tolère pas la moindre faille, il subsiste sans le moindre grain de sable qui viendrait perturber cette « machine à objectivité ».

Notre hypothèse tient à considérer que cette pathologie de l'adaptation affecte de nombreux détenus. Elle caractérise ces situations de sujets qui ont réussi cet « impossible » qui consiste à s'adapter à l'univers carcéral. Ces détenus ont franchi la limite d'un monde dans lequel n'existe plus que le réel et présentent un refoulement réussi de la fonction de l'imaginaire. Ces détenus ne rêvent plus ou ne voient plus l'intérêt qu'ils pourraient avoir à s'intéresser à leur vie onirique et, si l'on analyse froidement la situation, il semble bien qu'ils aient « raison ». Le banal, avec son mode d'adaptation, est l'état de la raison pure, de la rationalité lucide qui ne cherche plus à se projeter dans l'avenir, ou en d'autres territoires qui tout simplement n'existent plus. 
Clément est un patient que l'on peut qualifier $d$ '" hyper-adapté ». Lorsqu'on interroge les gardiens de prison et autres membres du personnel, ils le décrivent volontiers comme un détenu "modèle ". Respectant parfaitement les règles, il présente en outre cette faculté d'apprendre parfaitement les phrases qu'il doit dire à son interlocuteur. Il répète souvent d'un entretien à l'autre des paroles que nous lui avons prononcées et démontre qu'il a cherché à les appliquer : "Je fais ce que l'on me dit de faire " nous dit-il fréquemment. Il estime que c'est en adhérant parfaitement à ces différents discours qu'il sera rapidement libéré, c'est $d u$ moins ce que sa mère nous confie à son propos. Mais son problème apparaît lorsque nous lui demandons, en nous gardant de lui donner notre point de vue qu'il s'empresserait de s'approprier, ce qu'il veut faire une fois libéré. Quels sont ses projets? Comment voit-il son avenir? Clément est incapable de répondre à cette question. Après un long silence, il nous dit : "Il faut que je me renseigne, il faut que je demande autour de moi. »

Il nous faudra plusieurs semaines pour nous rendre compte qu'il est incapable de confier une pensée ou un point de vue qui lui appartiendrait. Cette absence de subjectivité semble, d'une certaine façon, passer inaperçue lors de nos entretiens car Clément est comme transparent et ne se fait donc pas remarquer. Un gardien nous dira un jour : "Il se fond tellement dans la masse qu'on a l'impression qu'il fait partie des murs, qu'il est comme certains lézards, il prend la couleur de son environnement. "Cette "transparence " qui agit comme un masque le rend "imperceptible ». Le problème est que le futur semble absent de l'existence de Clément. Si son attitude convient fort bien au quotidien carcéral, elle tracasse l'ensemble des professionnels de la prison qui ne peuvent se représenter Clément dans une vie différente, une vie dans laquelle il devrait produire, outre la productivité pour elle-même, de la subjectivité.

On pourrait suggérer que le sujet présente peutêtre, à travers cette attitude d'hyper-adaptation et de perte de subjectivité, une stratégie utile et, qu'une fois libéré, les mécanismes de subjectivation, de territorialisation, et de façon globale l'imaginaire réapparaîtront. C'est sûrement le cas pour certains mais nous pouvons penser également que bon nombre de ceux-ci éprouveront de grandes difficultés d'adaptation à une réalité extérieure qui, elle, demande toujours à être apprivoisée et marquée par des actes de modification que le détenu a «appris » à ne plus poser.

L'imaginaire en prison est donc frappé par un réel prenant toute la place et déterminant un sujet à s'exprimer dans le banal et à présenter une pathologie de l'adaptation, reposant sur un double paradoxe. D'une part, nous sommes confrontés à un ersatz d'adaptation; d'autre part, nous avons affaire à une pseudo-pathologie. En effet, cette dernière semble « ne pas appartenir au sujet »; elle ne procède pas à la création de nouvelles normes (nous entendons ici le pathologique dans la lignée de Canguilhem). Le sujet s'adapte au même, c'est là sa dimension pathologique; il est, en quelque sorte, hors du processus pathologique qui le façonne. C'est donc bien la totalité de l'expérience du sujet qui est organisée de l'extérieur, y compris son expérience pathologique, le conduisant à incarner le rôle d' " un être unique en général » (Sami-Ali, 1980), un rouage parmi d'autres de la mécanique carcérale.

\section{BiBLIOGRAPHIE}

BACHELARD, G. 1942. L'eau et les rêves. Essai sur l'imagination de la matière, Paris, Librairie José Corti, 1993.

Bachelard, G. 1957. La poétique de l'espace, Paris, Puf, 2004.

Canguilhem, G. 1966. Le normal et le pathologique, Paris, Puf.

CHAR, R. 1946. Feuillets d'Hypnos, Paris, Gallimard, 2007.

ENGLEBERT, J. 2013a. Psychopathologie de l'homme en situation. Le corps du détenu dans l'univers carcéral, Paris, Hermann.

ENGLEBERT, J. 2013b. « Bachelard, Kuhn et Starobinski. Note sur une épistémologie masquée ", Le Cercle herméneutique, $\mathrm{n}^{\circ}$ 20-21, p. 159-170. 
ENGLEBERT, J. 2014. « La magie et la sorcellerie des visages comme socle anthropologique de la philosophie sartrienne », Études sartriennes, $\mathrm{n}^{\circ} 17-18$, p. 41-59.

EngleBert, J. ; Follet, V. 2016. Adaptation. Essai collectif à partir des paradigmes éthologiques et évolutionnistes, Paris, MJWF édition.

GaUthier, J.-M. 1993. L'enfant malade de sa peau, Paris, Dunod.

Merleau-Ponty, M. 1945. Phénoménologie de la perception, Paris, Gallimard.

SAMI-ALI. 1970. De la projection, Paris, Dunod.

SAMI-ALI. 1974. L'espace imaginaire. Paris, Gallimard.

SAMI-ALI. 1977. Corps réel, corps imaginaire, Paris, Dunod, 1998.

SAMI-ALI. 1980. Le banal, Paris, Gallimard.

SAMI-Ali. 1987. Penser le somatique: imaginaire et pathologie, Paris, Dunod.

SAMI-ALI. 1990. Le corps, l'espace et le temps, Paris, Dunod, 1998.

SAMI-ALI. 1997. Le rêve et l'affect. Une théorie du somatique, Paris, Dunod.

SAMI-ALI. 2003. Corps et âme. Pratique de la théorie relationnelle, Paris, Dunod.

SARTRE, J.-P. 1936. L'imagination, Paris, Puf, 2007.

SARTRE, J.-P. 1939. Esquisse d'une théorie des émotions, Paris, Hermann, 1995.

SARTRE, J.-P. 1940. L'imaginaire, Paris, Gallimard.

SARTRE, J.-P. 1943. L'être et le néant, Paris, Gallimard.

VIAN, B. 1947. L'écume des jours, Paris, Pauvert. 